\title{
Teaching Procedural Skills to Medical Students: A Pilot Procedural Skills Lab
}

\author{
Laurence M. Katz ${ }^{1,4}$, Alexander Finch ${ }^{1}$, Tyler McKinnish ${ }^{1}$, Kurt Gilliland ${ }^{2}$, \\ Sue Tolleson-Rinehart ${ }^{3}$, Bonita L. Marks ${ }^{1,4}$ \\ Departments of ${ }^{1}$ Emergency Medicine, ${ }^{2}$ Cell Biology and Physiology and ${ }^{3}$ Pediatrics, University of North Carolina School of Medicine, \\ ${ }^{4}$ Department of Exercise and Sports Science, University of North Carolina, Chapel Hill, NC, USA
}

\begin{abstract}
Background: Medical students have limited confidence in performing procedural skills. A pilot study was conducted to evaluate the effect of a multifaceted Procedural Skills Lab (PSL) on the confidence of medical students to perform procedural skills. Methods: Twelve $2^{\text {nd }}$ year medical students were randomly selected to participate in a pilot PSL. The PSL students met with an instructor for $2 \mathrm{~h}$ once a week for 4 weeks. Students participated in a flipped classroom and spaced education program before laboratory sessions that included a cadaver laboratory. Procedural skills included a focused assessment with sonography in trauma (FAST) scan, cardiac echocardiogram, lumbar puncture, arthrocentesis, and insertion of intraosseous and intravenous catheters. Students in the PSL were asked to rank their confidence in performing procedural skills before and after completion of the laboratory sessions (Wilcoxon ranked-sum test). A web-based questionnaire was also emailed to all $2^{\text {nd }}$ year medical students to establish a baseline frequency for observing, performing, and confidence performing procedural skills (Mann-Whitney U-test). Results: Fifty-nine percent $(n=106)$ of $1802^{\text {nd }}$ year medical students ( $n=12$ PSL students [treatment group], $n=94$ [control group]) completed the survey. Frequency of observation, performance, and confidence in performing procedural skills was similar between the control and treatment groups at baseline. There was an increased confidence level $(p<0.001)$ for performing all procedural skills for the treatment group after completion of the PSL. Discussion: An innovative PSL may increase students' confidence to perform procedural skills. Future studies will examine competency after a PSL.
\end{abstract}

Keywords: Flipped classroom, procedural skills, spaced education, undergraduate medical education

\section{Background}

Procedural skill training is an important component of medical education, but opportunities to learn and become competent in procedural skills during medical school are limited. ${ }^{[1]}$ Failure to learn procedural skills prior to residency may increase resident stress and medical errors arising from provider inexperience. ${ }^{[2,3]} \mathrm{A}$ trend in medical education is

\begin{tabular}{|l|l|}
\hline \multicolumn{2}{|c|}{ Access this article online } \\
\hline Quick Response Code: & Website: \\
\hline & www.educationforhealth.net \\
\cline { 2 - 2 } & \\
\hline
\end{tabular}

Address for correspondence:

Dr. Laurence M. Katz, Department of Emergency Medicine, University of North Carolina School of Medicine, 170 Manning Drive, Chapel Hill, NC 27599, USA.

E-mail: Ikatz@med.unc.edu to introduce clinical clerkships earlier into the curriculum to meet the current medical needs, but students in clinical clerkships tend to be delegated to passive roles rather than active participants. ${ }^{[4]}$ An innovative Procedural Skills Lab (PSL) early in the medical school curriculum may be of value in preparing medical students for mastery of certain procedural skills and Entrustable Professional Activities (EPAs) required for residents. ${ }^{[5]}$

Procedural skill training is usually hands-on and labor intensive. A number of teaching innovations may reduce that burden. A flipped classroom provides students with

This is an open access article distributed under the terms of the Creative Commons Attribution-NonCommercial-ShareAlike 3.0 License, which allows others to remix, tweak, and build upon the work non-commercially, as long as the author is credited and the new creations are licensed under the identical terms.

For reprints contact: reprints@ medknow.com

How to cite this article: Katz LM, Finch A, McKinnish T, Gilliland K, Tolleson-Rinehart S, Marks BL. Teaching procedural skills to medical students: A pilot procedural skills lab. Educ Health 2017;30:79-83. 
an opportunity to view instructional procedural skills videos at their convenience prior to laboratory sessions, increasing the efficiency of subsequent hands-on training sessions ${ }^{[6]}$ Spaced education is another teaching format that may compliment a hands-on PSL. ${ }^{[7]}$ Spaced education is a web-based, student-directed learning tool. Students are provided with a series of review questions available by email or electronic mobile application that challenges a student's knowledge of procedural skills. When a student answers a question incorrectly, he or she is given the correct answer and an explanation for it. The same question is sent back to the student at a later date. When the student provides the correct answer on consecutive testing sessions, the question is "retired" and a new set of questions is provided. Spaced education allows students to establish their own pace of learning while increasing the efficiency and retention of new knowledge.

The objective of this study was to determine the effect of an innovative PSL on medical students' confidence in performing procedural skills. We hypothesized that a PSL incorporating a flipped classroom, spaced education, cadavers, and simulation laboratories would improve students' confidence to perform procedural skills.

\section{Intervention}

The PSL met for $2 \mathrm{~h}$ once a week for 4 weeks. Students were asked to view NEJM procedural skills' videos (http://www. nejm.org/multimedia/medical-video) prior to laboratory sessions. Students were also provided with access to a commercially available spaced education program (QStream, Burlington, MA, USA). A single instructor supervised students individually as they practiced each procedural skill in $10 \mathrm{~min}$ intervals. Students were paired for ultrasound sessions, with students taking turns acting as models for each other. The remaining members of the laboratory surrounded the student ultrasonographer to share in discussions with the instructor. Lumbar punctures, arthrocentesis, and intraosseous insertion were taught and practiced on cadavers in the cadaver laboratory. Insertion of intravenous (IV) catheters was practiced on a simulation arm prior to students pairing to practice on each other in the simulation laboratory.

\section{Methods}

\section{Institutional Review Board and participants}

The study was approved by the University of North Carolina at Chapel Hill Institutional Review Board. All $1802^{\text {nd }}$ year medical students were invited by email to volunteer for participation in a PSL. Forty students responded to the invitation and 12 students were randomly chosen through a lottery system for participation in the PSL.

\section{Study design}

After the PSL had concluded, we performed a cross-sectional study by sending an anonymous web-based survey by email to all the $2^{\text {nd }}$ year University of North Carolina medical students. We created the survey using Qualtrics survey software (Qualtrics, Provo, Utah, USA) to measure students' frequency of observation and performance of procedural skills. The procedural skills were a FAST scan, lumbar puncture, knee arthrocentesis, intraosseous catheter placement, cardiac ultrasound, and IV catheter insertion. We asked students to assess their confidence in performing procedural skills on a Likert scale that ranged from $1=$ not confident at all to $4=$ very confident. Students who had not participated in the PSL were designated as the control group. The students who participated in the PSL (treatment group) were asked to report the frequency of observation/performance of procedural skills and their confidence in performing procedural skills before and after participation in the laboratory sessions.

\section{Data analysis}

We used the Mann-Whitney U-test to determine the differences in observation, performance, and confidence in performing procedural skills between the treatment group (pre-PSL) and control group. We used the Wilcoxon signed-rank test to evaluate the change in the confidence of students to perform procedural skills pre- and post-participation in the PSL. We set significance at $p<0.05$ and analyzed the data with SPSS software, Version 23 (IBM, Armonk, NY, USA).

\section{Results}

One hundred and six (59\%) of $1802^{\text {nd }}$ year medical students ( $n=12$ PSL participants [treatment group], $n=94$ [control group]) completed the survey. The pretest frequency of students' observation of procedural skills was similar between the control and treatment groups [Figure 1]. Aside from inserting IV lines, the majority of participants in both groups had not observed procedural skills by the time of the pretest survey. With the exception of cardiac echocardiogram ( $U=488, Z=-2.064, p=0.039)$, the pretest frequency of performing procedural skills was similar between control and treatment groups [Figure 2]. At the time of the pretest survey, the majority of students had not performed procedural skills included in the survey. Before the PSL, students' confidence in their ability to perform procedural skills was similar between the control and treatment groups [Figure 3]. Students who participated in the skills laboratory reported a significant increase in confidence in their ability to perform procedural skills for all procedures post-treatment [Table 1], and the change in pretest distribution of confidence levels for each procedure is shown in Figures 4-9. 


\section{Discussion}

The majority of the $2^{\text {nd }}$ year medical students at the University of North Carolina School of Medicine have neither observed nor performed common procedural skills. Therefore, it is not surprising that students are not confident that they can perform these procedures. Dehmer et al. found a similar lack of confidence even in the $4^{\text {th }}$ year medical students' performance of basic procedural skills. ${ }^{[1]}$ Now that clerkships are occurring earlier in the medical school curriculum, introducing an innovative PSL early in the curriculum may be valuable. A PSL early in the curriculum may provide students with the opportunity to

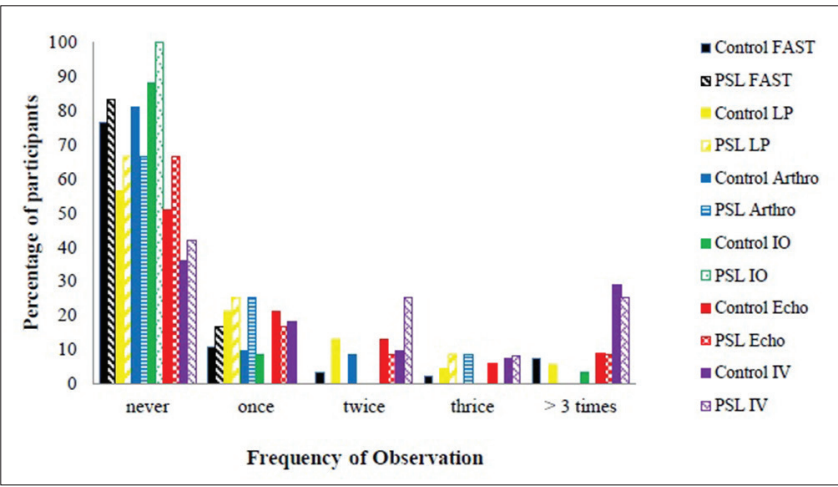

Figure 1: Frequency for observing procedural skills at baseline. PSL=Procedural Skills Lab, FAST=Focused assessment with sonography in trauma, LP=Lumbar puncture, Arthro=Arthrocentesis, $\mathrm{IO}=$ Intraosseous, Echo=Cardiac ultrasound, IV=Intravenous

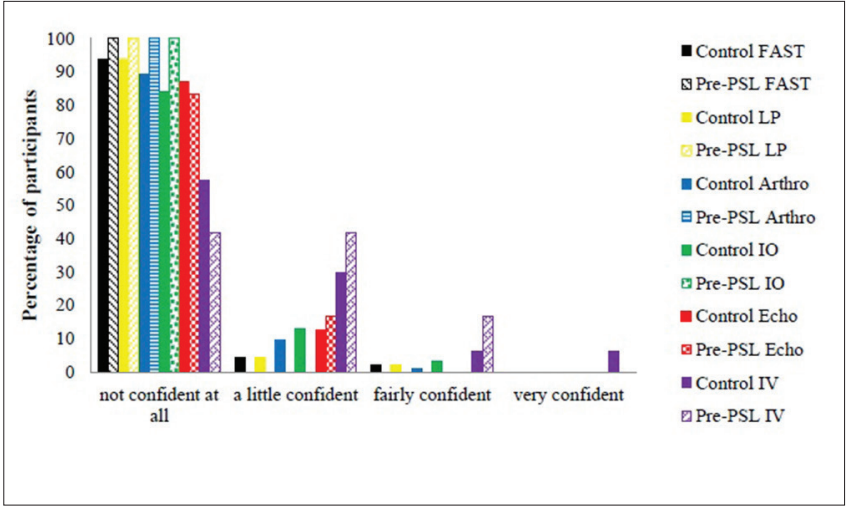

Figure 3: Confidence in performing procedural skills at baseline. $P S L=$ Procedural Skills Lab, FAST=Focused assessment with sonography in trauma, LP=Lumbar puncture, Arthro=Arthrocentesis, $\mathrm{IO}=$ =Intraosseous, Echo=Cardiac ultrasound, IV=Intravenous practice and become confident in performing procedural skills while addressing multiple EPAs as recommended by the Association of American Medical Colleges before graduation..$^{[5,8]}$

The PSL incorporated a flipped classroom, spaced education, cadaver laboratory, and a simulation environment to create an engaging and efficient approach for students to learn and practice procedural skills. The flipped classroom model allowed students to become familiar with the practical aspects of performing procedural skills without the need for faculty or classroom resources. ${ }^{[6]}$ Review questions presented in a spaced education format complemented the

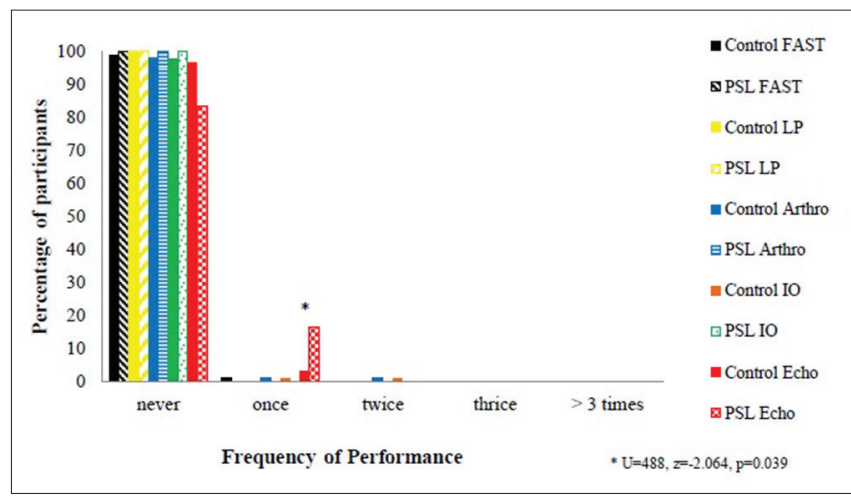

Figure 2: Frequency for performing procedural skills at baseline. PSL=Procedural Skills Lab, FAST=Focused assessment with sonography in trauma, LP=Lumbar puncture, Arthro=Arthrocentesis, $\mathrm{IO}=$ Intraosseous, Echo=Cardiac ultrasound

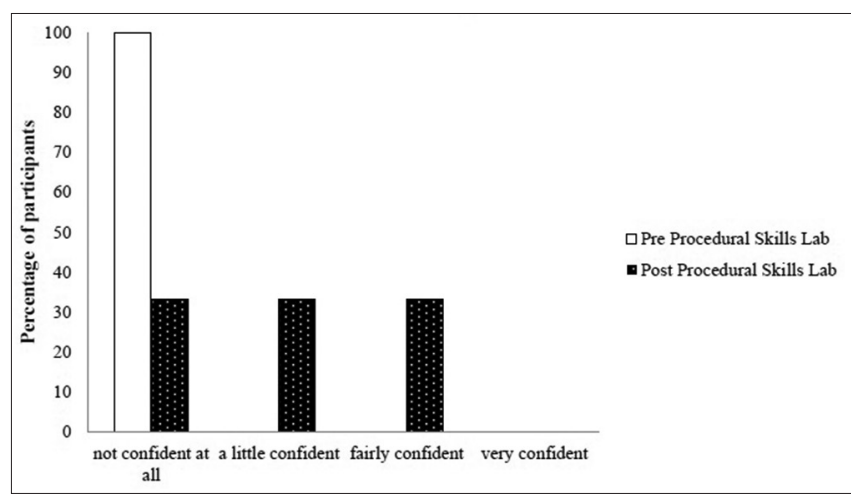

Figure 4: Confidence in performing a FAST scan before (pre) and after (post) participation in the Procedural Skills Lab. FAST=Focused assessment with sonography in trauma

\begin{tabular}{lcccc}
\hline Table 1: Student confidence performing procedural skills pre and post participation in the procedural skills lab \\
\hline Procedural Skill & Confidence Pre (median) & Confidence Post (median) & Effect size $(\boldsymbol{r})$ & $Z$ \\
\hline FAST scan & 1 & 2 & 0.53 & -2.59 \\
Lumbar puncture & 1 & 2 & 0.62 & -3.02 \\
Arthrocentesis & 1 & 3 & 0.62 & -3.04 \\
Intraosseous catheter insertion & 1 & 3 & 0.61 & -2.97 \\
Cardiac ultrasound & 1 & 2.5 & 0.53 & -2.59 \\
Intravenous catheter insertion & 1 & 3 & 0.65 & -3.010 \\
\hline
\end{tabular}




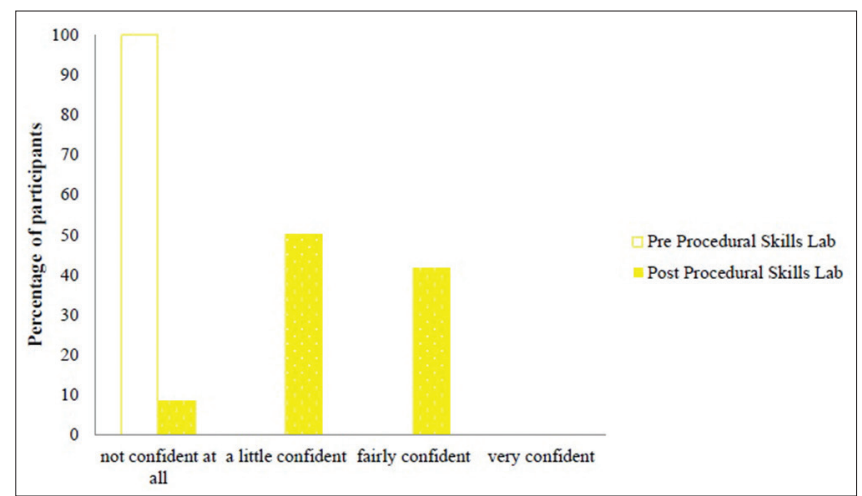

Figure 5: Confidence in performing a lumbar puncture before (pre) and after (post) participation in the Procedural Skills Lab

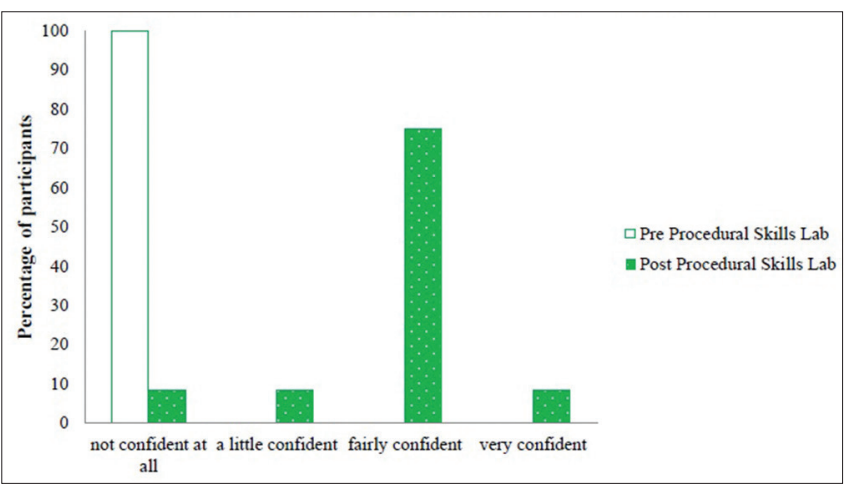

Figure 7: Confidence in inserting an intraosseous catheter before (pre) and after (post) participation in the Procedural Skills Lab

procedural videos and assisted students with development of the theoretical foundation for procedural skills. The rate of learning was guided by the students and was reported to be highly motivational. ${ }^{[7]}$ The simulation laboratory allowed students to practice multiple procedural skills using a team approach (e.g., one student established IV access while another performed cardiopulmonary resuscitation on the same manikin). The cadaver laboratory created a reverent human experience for performing procedural skills without putting patients at risk. Students also volunteered to allow other students to practice on them with bedside ultrasound and IV insertion, providing further insight into the patient experience. These multimodal learning experiences engaged students to develop confidence in performing procedural skills through hands-on learning which is not always possible in clinical clerkships. ${ }^{[4]}$

This study had a number of limitations. First, the study was retrospective, using a self-reporting format possibly accompanied by recall bias. However, the study was performed within a year of completing the pilot laboratory, so recall should be reasonably accurate. ${ }^{\left[{ }^{[9}\right.}$ Second, the study may have had some selection bias, as highly motivated students may have been more likely to respond to the

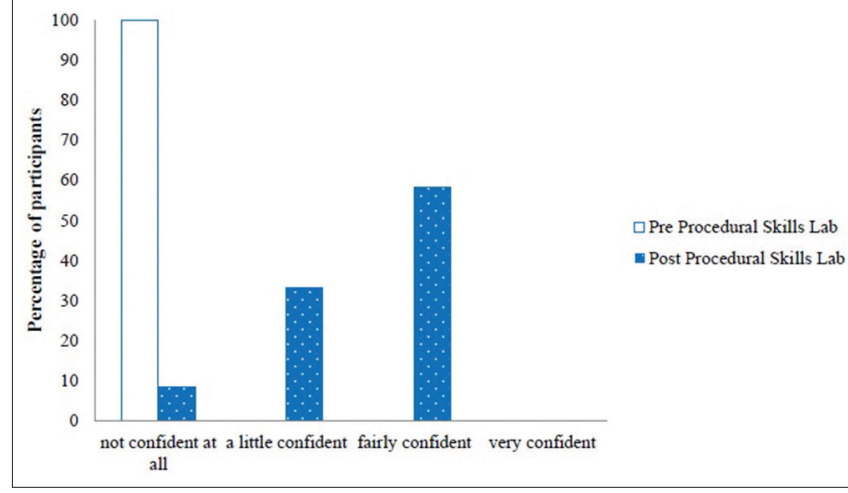

Figure 6: Confidence in performing an arthrocentesis before (pre) and after (post) participation in the Procedural Skills Lab

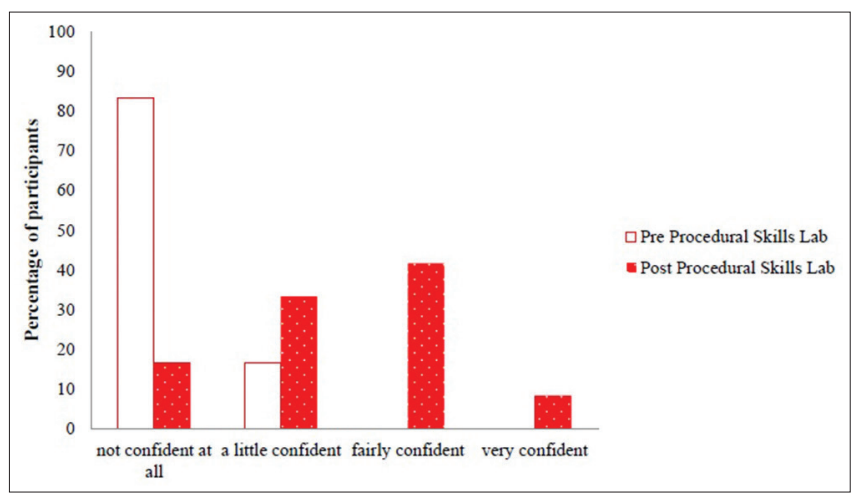

Figure 8: Confidence in performing a cardiac ultrasound before (pre) and after (post) participation in the Procedural Skills Lab

recruitment invitation to participate in the pilot PSL. Selection bias was mitigated, to the best of our ability, by incorporating a lottery randomization procedure for selection into the treatment group. Comparable baseline procedural skills experience and confidence for performing procedural skills between the control and treatment groups before completing the laboratory also suggested low selection bias. Third, this pilot PSL could only accommodate 12 students. The small sample size and single institution site could affect the generalizability of the results. Even with this small number of students, pre-post changes in confidence levels were statistically significant. Finally, we did not use measures of procedural competence or other assessments to evaluate students in the PSL. A follow-up prospective study is planned using the PSL with evaluation of competence and comparing other types of procedural learning.

\section{Discussion}

Second-year medical students may have limited experience with, and confidence in, performing procedural skills. An innovative PSL may impart valuable experience and increase students' Confidence in performing procedural skills. 


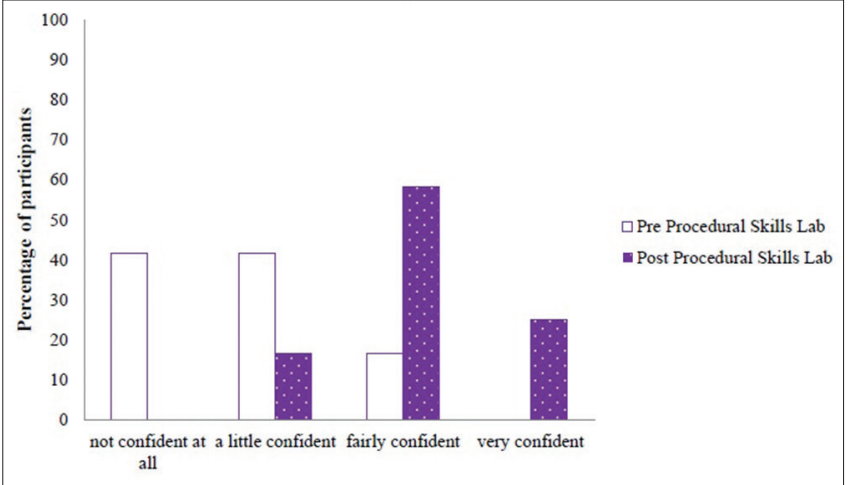

Figure 9: Confidence in inserting an intravenous catheter before (pre) and after (post) participation in the Procedural Skills Lab. PSL=Procedural Skills Lab

\section{Acknowledgments}

The authors would like to thank the $2^{\text {nd }}$ year medical students of the University of North Carolina School of Medicine for their participation and support.

\section{Financial support and sponsorship}

Nil.

\section{Conflicts of interest}

There are no conflicts of interest.

\section{References}

1. Dehmer JJ, Amos KD, Farrell TM, Meyer AA, Newton WP, Meyers MO. Competence and confidence with basic procedural skills: The experience and opinions of fourth-year medical students at a single institution. Acad Med 2013;88:682-7.

2. Ziv A, Wolpe PR, Small SD, Glick S. Simulation-based medical education: An ethical imperative. Acad Med 2003;78:783-8.

3. Promes SB, Chudgar SM, Grochowski CO, Shayne P, Isenhour J, Glickman SW, et al. Gaps in procedural experience and competency in medical school graduates. Acad Emerg Med 2009;16 Suppl 2:S58-62.

4. Remmen R, Denekens J, Scherpbier A, Hermann I, van der Vleuten C, Royen PV, et al. An evaluation study of the didactic quality of clerkships. Med Educ 2000;34:460-4.

5. AoAMC. Core Entrustable Professional Activities for Entering Residency; 2015. Available from: https://www.aamcorg/cepaer. [Last accessed 2016 Apr 02].

6. McLean S, Attardi SM, Faden L, Goldszmidt M. Flipped classrooms and student learning: Not just surface gains. Adv Physiol Educ 2016;40:47-55.

7. Long A, Kerfoot BP, Chopra S, Shaw T. Online spaced education to supplement live courses. Med Educ 2010;44:519-20.

8. Learning objectives for medical student education - Guidelines for medical schools: Report I of the Medical School Objectives Project. Acad Med 1999;74:13-8.

9. Kupek E. Bias and heteroscedastic memory error in self-reported health behavior: An investigation using covariance structure analysis. BMC Med Res Methodol 2002;2:14. 
(c) 2017. This work is published under

https://creativecommons.org/licenses/by-nc-sa/4.0/(the "License").

Notwithstanding the ProQuest Terms and Conditions, you may use this content in accordance with the terms of the License. 